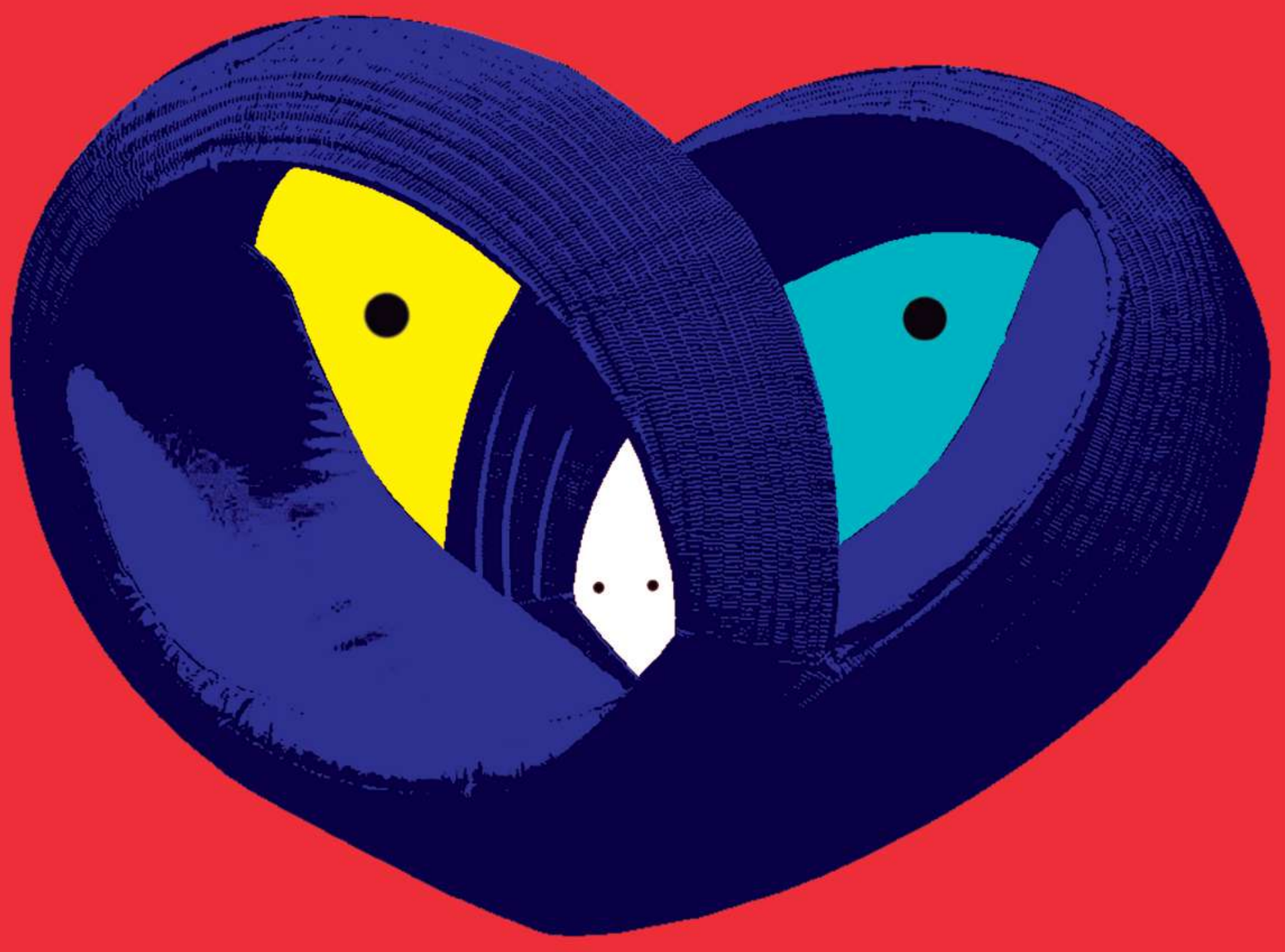


Colaboración

entre artesanos

y diseñadores

mexicanos:

en busca

de nuevos signos

Alejandra Rojo Cabrera 


\section{Colaboración entre artesanos y diseñadores mexicanos: en busca de nuevos signos}

\section{Alejandra Rojo Cabrera}

rojo.alejandra@gmail.com | Diseñadora de productos y espacios. Profesora de design thinking e historia y metodología del diseño. Coach de creatividad en Dalia Empower.

Fecha de recepción: 21 de julio de 2017 | Fecha de aceptación: 05 de abril de 2018

\section{Resumen}

Este documento presenta un acercamiento a las relaciones que se establecen entre artesanos y diseñadores, enfocándose en el intercambio de conocimientos y el proceso de aprendizaje que se desprende, así como las funciones que ambos desempeñan en proyectos colaborativos. Después se presentan cuatro proyectos contemporáneos, el aprendizaje sobre el manejo de las relaciones que han establecido y los retos que han enfrentado al facilitar estas experiencias de intercambio de conocimientos.

Por último, se muestra un panorama sobre los aspectos que intervienen en la construcción de un lenguaje contemporáneo en el diseño mexicano y el papel del diseñador en este proceso. Se responde a interrogantes sobre la identidad y características del diseño mexicano contemporáneo. Finalmente, el documento retoma la importancia de las exposiciones como un modo de difusión del diseño.

Palabras clave | diseño, artesanía, colaborativo, mexicano, identidad 


\section{Abstract}

This paper presents an approach to the relationships established between artisans and designers, focusing on the exchange of knowledge and the learning process that emerges, as well as their roles in collaborative projects. Then presents four contemporary projects, the learning about the management of the relationships established, and the challenges that have faced to facilitate these experiences arise from Exchange of knowledge.

Finally, shows a picture of the aspects involved in the construction of a contemporary Mexican design language and the role of the designer in this process, responding to questions about the identity and characteristics of modern Mexican design. Finally, the document takes up the importance of exhibitions as a way of dissemination of design.

Keywords | design, crafts, collaborative, mexican, identity. 


\section{Introducción}

En este documento se busca entender cómo se construyen las relaciones y el aprendizaje que ocurre entre diseñadores y artesanos a través de los proyectos que desarrollan en común; para comprender estos temas desde un punto de vista actual y activo, se realizó un cuestionario a cuatro proyectos con diversos propósitos pero que comparten una relación cercana con artesanos, el primero fue el extinto Centro de diseño de Oaxaca elegido por desarrollar una metodología para poder implementar proyectos de trabajo entre diseñadores y artesanos del estado de Oaxaca, el segundo es The chair that rocks, el cual fue elegido por facilitar experiencias de aprendizaje entre alumnos de diseño y artesanos, el tercero Rituales contemporáneos por trabajar con pequeños talleres de manera local, es decir, situados en la Ciudad de México y el cuarto Víctor Alemán por fusionar procesos de desarrollo digital con el trabajo artesanal.

Estos cuestionarios plantearon las mismas interrogantes para todos, con los cuales se pretende entender sus motivaciones, retos, consejos y aprendizajes a través de sus proyectos, pero también se hacen cuestionamientos sobre el trabajo del diseñador como intérprete de la tradición, su punto de vista sobre la construcción de una identidad propia en el diseño mexicano y ejemplos que les resultarán destacados en esta materia; otro de los puntos es si consideran que su educación como diseñadores industriales es la que les permite poder realizar estos cambios que logran mediante su trabajo.

El propósito de esta exploración es comprender el impacto de los diseñadores en la construcción de la identidad del diseño mexicano: si es resultado de una postura consciente o si emerge de una manera implícita en su trabajo.

El crear condiciones de vida favorables facilita que los miembros de una sociedad puedan cubrir sus necesidades, sin embargo, cuando esto no pasa, el resguardo de conocimientos ancestrales se pone en peligro al no ser prioridad. No importa la calidad, ni la destreza manual que tenga un artesano mientras no exista un mercado al cual pueda vender sus piezas, por lo tanto sus recursos serán insuficientes. De igual manera que los esfuerzos por parte 
de las instituciones educadoras en materia de diseño se pueden ver mermados al enfrentar a sus graduados a un mercado laboral en el cual aún existe un desentendimiento de su disciplina, mediante comportamientos como la reducción de la actividad a un nivel técnico o la inclinación por hacer copias del diseño producido en otros países.

Para poder aspirar a un crecimiento en estas áreas, se necesita entender la situación por la que se transita, para así poder tomar acciones que cooperen con el desarrollo de condiciones para realizar cambios. Un primer acercamiento a esto sería contar con un plan estratégico que unificara los programas de apoyo y difusión, así como la coherencia en los discursos políticos, culturales y de promoción turística, los cuales tienden a exaltar la diversidad y la calidad del trabajo de los artesanos mexicanos, como parte de la ponderada cultura del país, no obstante, en muchos casos, la realidad es que aquellos que elaboran esos trabajos que tanto orgullo inspiran, aún siguen experimentando condiciones de vida poco favorables que atentan a la continuidad de su labor.

De acuerdo con el Fondo Nacional para el Fomento de las Artesanías (FONART, 2009) uno de los problemas más difíciles de afrontar para la comunidad artesanal es la transmisión intergeneracional de la pobreza. Un alto porcentaje de este sector de la población forma parte de las 607 mil personas que se encuentran por debajo de la línea de bienestar (indicador de pobreza), aunado a una alimentación deficiente y la carencia de seguridad social la cual recientemente ha sido atendida a través de la inclusión de los artesanos al seguro popular.

Las condiciones laborales son otro pendiente, por ejemplo el empleo secundario más común al que recurren los artesanos es el cultivo de maíz y frijol, le siguen el de vendedor ambulante, ayudante y guardia (FONART, 2009). Por estos factores, la artesanía, más que un sustento familiar, se ha convertido en un complemento para la economía familiar. La pérdida del conocimiento del oficio artesanal decae ante la falta de ganancias constantes, carencia que no permite que se supere la pobreza, por lo que va pasando de generación en generación. 
Estas dificultades son atendidas por el FONART mediante programas sociales y vertientes de atención, cuyos objetivos son la conservación de la identidad, la tradición creativa y los valores que se reflejan en estas expresiones. La idea es que a través de sus creaciones los artesanos puedan generar su propia independencia económica mediante la capacitación, asistencia técnica, apoyo a la producción, adquisición de artesanías, apoyos a la comercialización y la organización de concursos de arte popular.

Dentro del Plan Nacional de Desarrollo 2013-2018 del gobierno actual, es prioridad el desarrollo de sectores estratégicos en el país, donde el FONART debe cumplir con promover una visión emprendedora para desarrollar proyectos productivos rentables. Dejar el enfoque asistencialista es uno de los propósitos de la institución, sin embargo, la estrategia es alcanzar esto a través de apoyos económicos que incrementen los ingresos en la población. Para tener accesos a la ayuda los artesanos deben presentar una serie de documentos para que sus proyectos sean seleccionados por el Comité de Validación y Aprobación de Proyectos Artesanales (COVAPA). Estas ayudas se pueden utilizar con diversos propósitos, por ejemplo, se le pueden prestar 2 mil pesos para registrar la marca, 5 mil pesos para el diseño de un logotipo o 4 mil pesos para la certificación de normas oficiales mexicanas.

Las estrategias para solucionar el tamaño del problema aún son limitadas, los esfuerzos aislados de instituciones y los cambios de planes conforme el gobierno del momento, hacen que el impacto se reduzca. Lo que el FONART ha tratado de realizar es coordinar su trabajo con gobiernos estatales y centros de educación superior, un ejemplo es la atención a cinco grupos artesanales mediante un modelo de incubadora para fomentar el auto empleo; se les capacitó sobre la formación de un negocio, se realizó una exposición y se construyó un nodo de concentración de artesanías, así como asesoramiento legal para el registro de marcas. Este tipo de asesorías son una medida ante la poca rentabilidad de muchos de los proyectos artesanales. El problema tiene que ver con que las piezas son vendidas a precios bajos en comparación al tiempo invertido por el artesano, por lo que no se hace un cálculo adecuado del valor económico real. Nada fuera de lo que vive un diseñador, que se encuentra bajo la incertidumbre de no saber cuánto cobrar por un proyecto, habrá quien lo 
calcule en función del tiempo invertido, aunque castigue la productividad, otros por el tipo de cliente o por la explotación que harán del bien que se ha diseñado.

\section{El proceso de intercambio de conocimientos entre diseñadores y artesanos}

Si bien artesanos y diseñadores transitan situaciones similares, el complemento de sus habilidades y conocimientos, representa una alternativa viable y eficaz cuando comparten el objetivo de mejorar productos para tener una mayor aceptación en el mercado al cual se dirigen. Derivado de la experiencia propia para llegar a una situación en la que se propicie un intercambio de conocimientos, previamente tuvo que existir un acercamiento de reconocimiento mutuo, en el cual tanto el diseñador como el artesano se hayan identificado de tal manera que comience a existir una base de respeto recíproco por la actividad del otro. Todo esto bajo la suposición de querer realizar un trabajo colaborativo; en el caso que sea sólo por el interés de realizar la producción de un objeto, la relación dejaría de ser horizontal y estaría totalmente enfocada en los resultados que esta unión temporal traiga para ambos. En cualquiera de los casos se desprenderá un conocimiento. De una relación con propósitos colaborativos, el intercambio será mucho más intenso, habrá un entendimiento a profundidad del trabajo, tradición y técnicas, así como las condiciones alrededor de todo el proyecto para que éste resulte benéfico. De una relación por intereses productivos queda a decisión y curiosidad del diseñador los conocimientos que absorba por parte del artesano, al ejercer un rol de cliente es quien decide qué tanto se involucra.

Un proceso colaborativo a largo plazo cambiará la perspectiva y la actividad de los involucrados, surgirán resultados tangibles en el mejoramiento del producto final y eventualmente en las condiciones de vida. Cuando la relación está basada en la fabricación a pedido o la comercialización de los productos del artesano, la relación permanecerá, lo mismo que el beneficio económico para ambos, se tocarán temas administrativos y de eficiencia productiva, por lo que la experiencia será limitada; el diseñador tendrá como objetivo obtener el mejor provecho del trabajo del artesano y a su vez el artesano buscará mejorar sus tiempos de producción para satisfacer la demanda. 
Existen casos en los que los acuerdos se establecen de manera explícita, por ejemplo, Adelia Borges (2011) explica cómo se dieron las condiciones detrás de la famosa silla Multidao (2002) de los diseñadores brasileños conocidos como Los Hermanos Campana. La silla con una estructura metálica de acero inoxidable, cuyo asiento y respaldo está formado por una conjunción de muñecas de trapo producidas en Paraíba (localidad que no fue visitada por los diseñadores), de este tipo de sillas se hicieron series de pocas piezas a un alto costo, ganancias que se logran gracias a que los Campana son diseñadores reconocidos mundialmente, pero que nunca se hubieran alcanzado si la propuesta hubiera sido ideada por diseñadores sin prestigio o si viniera de una simple creación local de los artesanos.

La capacidad que tenga un individuo para adaptarse a participar en colaboraciones momentáneas y trabajo transitorio es muy valuada actualmente. Esto puede ser parte de la dicha y del pesar de hacer diseño. La figura del diseñador ha aparecido cada vez más como un agente esporádico, fuera de la nómina esencial en empresas, por lo que su trabajo es solicitado de manera independiente para solucionar problemas o cubrir necesidades; siempre pasando de un cliente a otro. Los artesanos se encuentran en una situación similar, pasan de un proyecto a otro, de un programa de apoyo a otro. $Y$ desde estas situaciones experimentadas por ambos, puede crecer la empatía de saberse un pensador por vocación, valuado muchas veces por sus cualidades de hacedor al servicio de un tercero.

Esta red de vínculos débiles, es lo que Richard Sennett (2000) llama la corrosión de la confianza, el compromiso mutuo y por lo tanto del carácter. Lo que él llama la cooperación artificial, de ahí la pregunta que él se hace ¿Cómo puede un ser humano desarrollar un relato de identidad e historia vital en una sociedad compuesta por episodios y fragmentos? Esto es la realidad de lo que se vive en oficinas y despachos, el diseñador va de una relación fugaz a otra, tratando de realizar el mejor trabajo posible, que le permita en un futuro próximo alcanzar una nueva conexión. 
Esta ansiedad cotidiana flexibiliza las circunstancias laborales, por lo que para adaptarse se necesita hacer uso de recursos internos como la autodisciplina, la cual resulta fundamental para todo diseñador y artesano al trabajar de manera independiente. Para integrarse al trabajo en equipo Sennett (2000) considera necesario desarrollar capacidades blandas, como ser un buen oyente, dispuesto a la cooperación y a la continua adaptación, aunque sea obligatoria y momentánea. Bajo estos términos plantea que el trabajo en equipo puede convertirse en la práctica en grupo de la superficialidad, aunque también es una oportunidad de receptividad mutua, y precisamente de eso trata el intercambio de conocimientos.

Lo anterior coincide con otra situación normalizada en el presente, en la cual se pueden encontrar personajes que hablan, discuten e incluso definen el futuro del diseño en el país, sin siquiera haberlo ejercido jamás; de la misma manera que se pueden encontrar administrativos que desarrollan planes y sistemas de merecimiento para los artesanos. Esta situación puede ser un tanto frustrante tanto para diseñadores como artesanos.

\section{La importancia de la intervención del diseñador}

A pesar que para llegar a ser diseñador desde una perspectiva formal académica, se requiere de una educación profesional, gran parte de los conocimientos que va adquiriendo un diseñador son producto de su curiosidad, de una disciplina autodidacta que le permite llegar a trazar su propia especialización. Resulta imposible pretender que todo diseñador sabe sobre cualquier tema que abarque su profesión y mucho más riesgoso asumir que lo sabe bien. De igual manera ocurre cuando un artesano toma conciencia de su labor, apoyado en sus conocimientos, se aventura a experimentar con nuevos materiales y procesos que reinventarán su oficio. La búsqueda entre ambos es similar y se realiza alrededor de elementos y recursos muy semejantes, de ahí que el entendimiento entre los dos debería ser más tácito.

El diseñador llega a entrometerse en una realidad ajena dentro de la cual la intención es hacer equipo, aunque su posición inevitablemente sea la de intermediario, no en el sentido de aprovechamiento desleal del microambiente y los conocimientos del artesano, sino que 
une entornos, culturas y personas. Esta especie de interfaz en la que se convierte el diseñador facilita la comunicación pero sobre todo la interacción de los productos del artesano con nuevos consumidores. El diseñador debe ser sensible a las condiciones, empático con el trabajo del artesano y astuto para encontrar los puntos a mejorar de tal manera que ponga a prueba las habilidades del artesano como un reto consigo mismo, utilizando todas sus cualidades para idear un plan que coloque a los productos en manos de los consumidores.

Parte de lo que puede aportar un diseñador al artesano es el pensar el diseño como un método para resolver problemas. Este entendimiento es un aprendizaje que tendrá repercusiones en la medida en la que el artesano comience a aplicarlo para cuestiones sobre cómo mejorar o qué cambios hacer, por lo que no sólo logrará la transformación de los objetos que produce, sino una modificación en la manera en que viene trabajando. Los conocimientos sobre procesos y control de calidad de un diseñador le permiten realizar cambios en el esquema organizacional, estructurando el trabajo y realizando filtros de calidad. Esta depuración de procesos mejorar la eficiencia y la calidad de los productos. Cuando un diseñador se familiariza con los materiales y sus transformaciones entiende el potencial que se puede alcanzar, esto puede llevar al desarrollo de nuevas formas que mejoren la función del objeto o que lo hagan interesante en términos estéticos por más por insignificantes que parezcan. El análisis de patrones y colores, que provienen de la naturaleza local o de la inspiración de elementos de la cultura, hace que el diseñador comprenda las constantes dentro de las referencias formales del artesano.

El diseñador está entrenado para saber cuáles son las cualidades más distintivas del producto, sabe cómo destacarlas y comunicarlas; parte de los desafíos que puede afrontar es influir en las técnicas y tecnologías de producción sostenibles, así como en la mejora de la administración del proyecto mediante la implementación de una estrategia de marca para los productos.

Algunos de los aspectos mediante los cuales el diseñador puede ayudar a que el producto del artesano sea mejor recibido en el mercado son a través de la información que se le 
provee al consumidor, comunicándole el origen, es decir, la localidad donde fue elaborado y las personas que contribuyeron a su producción, con el fin de que se tenga una perspectiva completa sobre el producto. Otras de las formas en las que el diseñador puede colaborar es simplemente mediante su trabajo, realizando logotipos, etiquetas explicativas, empaques, diseño del punto de venta, así como de alguna página web, de tal manera que el consumidor cuente con fuentes de información completa sobre lo que está adquiriendo.

Otro punto esencial es la documentación, el diseñador suele tener el vicio de no llevar un registro del desarrollo de sus productos o proyectos, por lo cual toda esa información valiosa se va perdiendo. Sin embargo el auge por el registro de los procesos ha hecho consciente al diseñador sobre la relevancia de documentar mediante fotografías, anotaciones, bocetos, planos y prototipos que ayudan a reconstruir el proceso, los cuales tienden a perderse en el ejercicio diario, y más cuando la actividad del diseñador y el artesano está sustentada en resultados tangibles. Como parte de la documentación de los proyectos el diseñador por su entendimiento formal puede ayudar al artesano a desarrollar representaciones visuales que le ayudan a entender lo que está haciendo y a comunicárselo a otros para ser capaz de desarrollar un lenguaje visual compartido.

\section{Proyectos contemporáneos de desarrollo y difusión del diseño entre artesanos y diseñadores en México}

Actualmente se pueden encontrar diversos proyectos de colaboración entre artesanos y diseñadores, de diversa índole, por ejemplo, como un referente de cambio en la visión del diseño a nivel estatal se encontraba en el Centro de Diseño de Oaxaca en este apartado se describe el punto de vista de Alejandra Villegas, en relación a la actividad que desempeñaba como responsable de proyectos en el Centro de diseño de Oaxaca. ${ }^{1}$ 


\section{El Centro de diseño de Oaxaca}

El Centro de Diseño de Oaxaca fue la primera institución pública promotora del diseño como herramienta estratégica en todo el país. Desde su creación en 2011 concretó más de 60 proyectos y 200 productos de diseño artesanal contemporáneo. Su equipo interdisciplinario trabajaba bajo las bases del diseño participativo a través de la vinculación con la sociedad civil, artesanos, artistas e instituciones educativas para desarrollar productos, servicios y experiencias para solucionar necesidades.

Dentro de los propósitos del centro, estaba el ser un lugar de encuentro y análisis para producir vínculos productivos, creativos y comerciales entre artesanos, diseñadores y artistas. El objetivo más importante era incidir de manera colaborativa en la creación de iniciativas y políticas públicas en las que el diseño fuera utilizado como un eje metodológico para mejorar las condiciones sociales, productivas y ecológicas del estado. Su contribución a la profesionalización y valoración del diseño los llevó a desarrollar uno de sus proyectos más destacados llamado Binomios Creativos dirigido por Alejandra Villegas, exdirectora de desarrollo de producto en el CDO. El programa vinculaba artesanos y diseñadores, además de un equipo de profesionales, para la implementación de procesos para desarrollar productos, impactando a las comunidades de manera positiva haciendo uso del diseño para generar desarrollo social.

Parte de la lucha que encabezaba el CDO, era en contra de la crítica de personas que partían de una base errónea al entender el diseño como un acto elitista que sólo crea sillas y lámparas de lujo, por lo que al compararlo con la pobreza existente en el estado lo consideran innecesario. Los proyectos que desarrollaba el centro buscaban favorecer la conformación de redes de apoyo basadas en la confianza y mediación. Para ellos en el proceso de intercambio de conocimientos entre un diseñador y un artesano es necesario formalizar las colaboraciones para que salgan a flote, es decir, firmar recibos y convenios para que las cosas queden claras por escrito, reafirmando el compromiso adquirido. 
Dentro de los proyectos que llevaban se dieron cuenta que el diseñador aprende a convivir con las diferencias entre él y el artesano, por ejemplo, los artesanos no acostumbran a registrar el desarrollo de su trabajo, por lo que no hay parámetros fijos de cantidades y tiempos; en estos casos el diseñador puede intervenir para que el artesano comprenda la importancia que tiene el registro para lograr una sistematización efectiva de su trabajo y medir sus alcances. El trabajo de Villegas en el proyecto Binomios Creativos consistía en procurar que los proyectos avancen, resolviendo obstáculos y adhiriendo a los equipos personas especializadas en temas particulares, además de facilitar el proceso de aprendizaje con los grupos de artesanos.

EI CDO creó una metodología para sus proyectos que se iba construyendo constantemente en función a los participantes, obstáculos, logros y avances del mismo. Para el centro el mayor aprendizaje que adquirían los diseñadores con los artesanos, era entender que hay otras realidades y que están bien así como son; necesitaban aprender a colaborar a pesar de las enormes diferencias económicas, educativas, de lenguaje, idiosincrasia, cosmovisión y ritmo de vida. En Binomios Creativos el diseñador no podía llegar a imponer sistemas, su función era entender y luego proporcionar ideas. Los artesanos por su parte aprendían la importancia que tiene la satisfacción de las necesidades del usuario y sus mercados.

Villegas considera que aprendió mucho sobre negociación y resolución de conflictos. Para que cada colaboración fuera exitosa se requería de integrar varios intereses y diseñar estrategias, era muy importante que los artesanos cambiaran sus paradigmas, de tal manera que les fuera más fácil comprender que estos esfuerzos eran encaminados a favor del desarrollo y la innovación, lo que los convierte en una inversión.

La experiencia fue lo que ha delimitado los lineamientos para los proyectos del CDO. Antes de elegir a las personas idóneas para los proyectos, se les preguntaba sobre sus motivaciones, se les especificaba el perfil y lo que tenían que hacer, es decir, sus responsabilidades; la estructura del trabajo horizontal no les había funcionado. Preferían que los objetivos quedaran acotados desde un principio para poder hacer el seguimiento y la 
evaluación necesarios, las reuniones sobre los proyectos tenían una estructura predefinida de actividades y acuerdos; los proyectos pasaban por distintos momentos, por lo que optaron por realizar contratos cortos y renovables, en caso de que se requiriera actualizar el equipo para no poner en peligro su seguimiento. Con el proyecto binomios creativos, Villegas buscaba que se entendiera cuál es la misión del diseño como disciplina, siendo el reto más importante generar estrategias de corresponsabilidad y autogestión, para que los artesanos se lo apropien.

Villegas encontró que el establecimiento de un código de principios colectivos ayudaba al equilibrio en las relaciones, cada quien entendía que el trabajo del otro es importante. Algo de lo que le tocó vivir era la desconfianza de los artesanos, ya que no se les convencía fácilmente de los beneficios de participar, uno de los argumentos en que incurrían era que el CDO se iba a aprovechar y acabaría vendiendo sus piezas en la ciudad de México ganándole mucho más. Para menguar esta desconfianza les explicaban el mecanismo de comercialización, para que no se sintieran explotados ni excluidos, la solución que encontraron fue una serie de esquemas gráficos en los que el artesano pudiera hacer un seguimiento de todos los pasos y personas involucradas que colaboraban para que sus piezas llegaran a las tiendas, y así entender el porqué de los costos y el precio final, al visualizar el trabajo que implicaba lograr. La transparencia en el proyecto es elemental para el desarrollo de confianza, a través de protocolos de información y funcionamiento que dan estructura a los objetivos, compromisos, tiempos, actividades y acuerdos económicos.

El CDO trabajaba en la construcción de una metodología aplicable y reguladora de los intercambios. En cambio, el proyecto de The chair that rocks sigue vigente, facilitando experiencias de conocimiento sobre todo a los diseñadores, a continuación se retoma parte de la información obtenida en el cuestionario enviado a José de la O sobre su experiencia. ${ }^{2}$ 


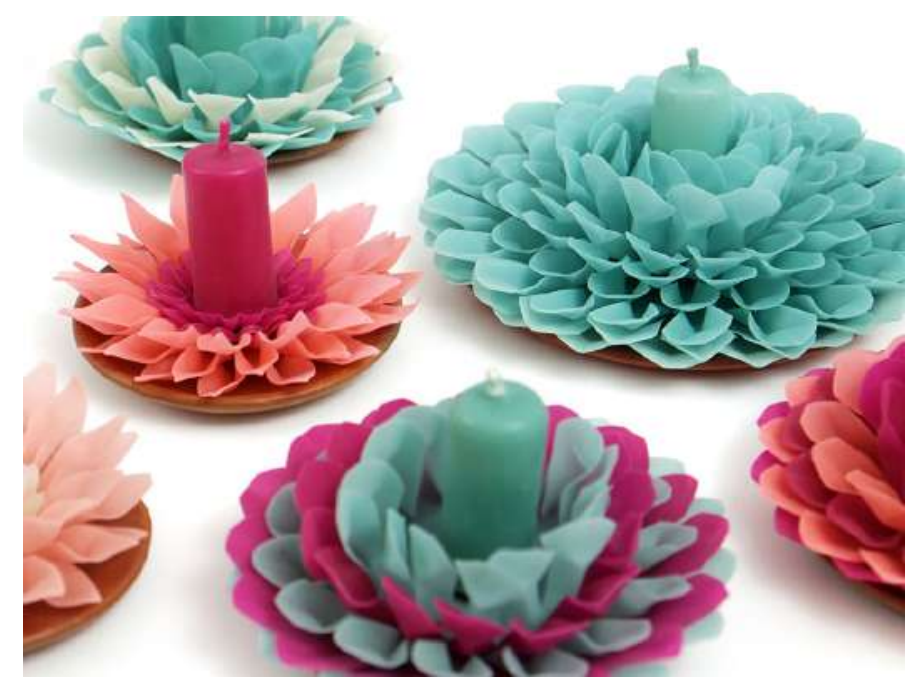

The Chair That Rocks

El objetivo del proyecto The chair that rocks según su creador el diseñador industrial José de la $O$ es vincular el diseño contemporáneo con los artesanos que se encuentran en Tlacotalpan, Veracruz. Se organizan experiencias en las que los diseñadores pasan unos días en la ciudad, donde diseñan y construyen un producto utilizando recursos locales y artesanías tradicionales. A través de este proyecto se pretende rescatar y preservar el trabajo artesanal local y sus técnicas, para reactivar la economía local. El propiciar estas experiencias hace que los diseñadores puedan aprender más sobre un nuevo oficio, producir e intercambiar conocimientos con los artesanos.

De la O, originario de Veracruz, desde pequeño estuvo en contacto con la región; su interés por Tlacotalpan va ligado a la diversidad de sus tradiciones culturales, él lo evoca como un lugar donde la gente toca instrumentos, baila, cocina e improvisa versos, además de los excelentes carpinteros creadores del famoso Sillón Tlacotalpeño o mecedora. Las sillas mecedoras se pueden ver en la entrada de cada casa y negocio del municipio, son una pieza de mobiliario indispensable espejo del estilo de vida del lugar; algunas otras de las artesanías locales son piezas cerámicas, ebanistería, la fabricación de dulces y la elaboración de instrumentos musicales a base de cuerdas. 
Una de las experiencias de aprendizaje que tienen los diseñadores en este proyecto, es un taller de siete días en el cual se rediseña y construye una silla inspirada en los diseños antiguos de cedro rojo y mimbre, todo esto se realiza en la carpintería Casa Prieto, un negocio familiar de tres generaciones a cargo del maestro carpintero Oscar Prieto. Por medio de esta experiencia el diseñador puede aportar algo diferente, desde un cambio mínimo que mejore su uso, hasta innovar con una propuesta de carácter metafórico inspirada en la localidad. Para poder ser parte de estas prácticas, a quienes aplican solicitud se les realiza una evaluación previa, el diseñador tiene que plantear por qué le interesa entrar al taller, qué es lo quiere aportar y cuáles son sus expectativas, esto con el fin de saber si es adecuado para la situación, de modo que vaya con una actitud empática.

Cuando comienza el intercambio de conocimientos ambos tienen que tener un objetivo en común antes de comenzar a trabajar; en el proceso las diferencias se acentúan y es probable que se den tensiones, las cuales a De la O le resultan entretenidas. Parte elemental del aprendizaje que adquieren los diseñadores es sensibilizarse al papel que les corresponde como aportadores de ideas, sin tratar de imponer metodologías. Si el diseñador quiere contribuir, inevitablemente tendrá que enfrentarse a un proceso de análisis para después mediante el diálogo lograr la aceptación de su propuesta. Aprender sobre el origen de los materiales le permite al diseñador valorar el fácil acceso que tiene a estos, por eso estas experiencias de aprendizaje comienzan en el caso de la carpintería, con el cepillado y canteado de un tablón en bruto pasando por conocimientos sobre el corte de la madera y cómo evitar el desperdicio del material.

Parte del aprendizaje se encuentra también en propiciar que el diseñador dialogue e incluso discuta con el artesano mientras fabrican una pieza, los dos tratan de mediar entre sus visiones, así es como según De la O se van ganando la confianza del uno al otro. En cambio, el aprendizaje de los artesanos es más difícil de identificar en este tipo de experiencias, ya que en el caso de los carpinteros ya tienen experiencia enseñando, les es muy natural. Para los artesanos la experiencia causa que vuelven a apreciar sus tradiciones y su oficio, De la O ha detectado el gusto porque gente de otros lados esté interesada en lo que ellos hacen, 
COLABORACIÓN ENTRE ARTESANOS Y DISEÑADORES

MEXICANOS: EN BUSCA DE NUEVOS SIGNOS

eso hace que quieran seguir siendo parte de esos proyectos. El facilitar estas experiencias para De la O es comparable con dar clases de diseño, ya que parte de sus funciones es impulsar a que los participantes hagan cosas innovadoras, ayudándoles a resolver problemas de diseño.

Como parte de la difusión del proyecto realizó un documental (The chair that rocks, 2013) donde se puede apreciar el contexto, el estilo de vida y el trabajo que realizan los carpinteros en Tlacotalpan como el desarrollo de las plantillas para los cortes, por ejemplo, en algunos casos imitan otras sillas o se basan en revistas y catálogos. Como parte de esto el Sr. Prieto, carpintero de oficio, hace una declaración muy interesante, en la cual dice que en Tlacotalpan todavía no ha habido el egoísmo de patentar algo. Esto confronta la visión que se tiene de la autoría, para De la $\mathrm{O}$ es algo de lo que los diseñadores deben aprender, ya que se le da mucha importancia al derecho de autor pensando que sus ideas son únicas, por lo que olvidan que toda buena idea acaba siendo apropiada por la sociedad.

Lo que se hace es replicar lo ya existente, tratando de mejorarlo con elementos mínimos, por lo que sería casi inmoral registrar los diseños que por historia le pertenecen a la comunidad. La rocking chair estándar que ofrece De la $\mathrm{O}$, es producida en un taller local, el reto más grande es la logística y mantener la silla en existencias con respecto a la demanda. Los artesanos muchas veces colapsan en estás etapas administrativas de los proyectos, así que De la $\mathrm{O}$ sugiere que el mejor consejo que le puede dar a un artesano que quiera mejorar la venta de sus productos, es atreverse a pedir ayuda, abrir una cuenta de correo electrónico que pueda atender, y facturar para que se profesionalice la visión que tienen de su trabajo. Por otro lado los artesanos que practican un oficio desde hace generaciones en la ciudad, tienen inconvenientes ligados al olvido y descalificación de su trabajo debido a nuevas tecnologías de producción. Existen proyectos como Rituales Contemporáneos que se han concentrado en esta revalorización, se les cuestionó sobre su proyecto e intenciones. ${ }^{3}$

3 Crf. Arzate, M. (23 de noviembre de 2014). Entrevista Tesis. (A. Rojo, Entrevistador).

Recuperado de: https://drive.google.com/open?id=1oeCiqZof1DISeSTQSNiWGaJ4EiDIFfPY 


\section{Enjoy tradition.}

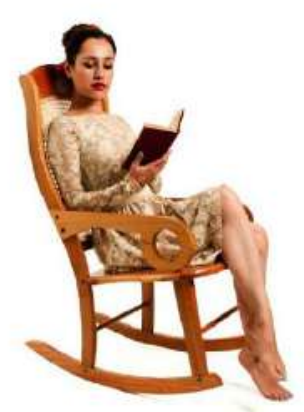

The Standard Rocking Chair

\section{Rituales contemporáneos}

Lo que motivó a que los diseñadores industriales Mariana y Gerardo Arzate dirigieran su práctica hacia el trabajo con los oficios tradicionales de la ciudad, fue el hecho de saber que existe una enorme cantidad de personas con un bagaje cultural técnico impresionante, que han preservado conocimientos que son parte de la cultura, historia e identidad local.

El requisito de mayor importancia en la concepción de sus productos es que estén bien pensados y por lo tanto bien diseñados para que puedan resolver necesidades cotidianas. Sus productos tienen un compromiso con el medio ambiente, tomando medidas como la reducción de material y la optimización de procesos que les permita elaborar objetos duraderos.

Ellos se suman a la comunidad colaborando y apoyando proyectos con productores mexicanos con el fin de revalorar las tradiciones y retomar los oficios; la base es conformar nuevas historias a partir de viejas técnicas para desarrollar objetos híbridos que incorporan tecnología actual con técnicas locales que les permitan elaborar pequeñas producciones. Esta mezcla de técnicas se puede apreciar en sus lámparas Luciérnagas de papel y en los ya clásicos contenedores para huevos Ilamados Corralitos y Gallineros. 
El trabajo que realizan ocurre en talleres pequeños con diversas técnicas como ebanistería, papel picado, torneado de madera, alambreros, orfebres, etc. Para poder realizar alianzas con estos talleres Mariana Arzate considera que el diseñador no debe mostrarse invasivo ni impositivo, al contrario, para establecer un intercambio de conocimiento fructífero, se debe hacer un buen diagnóstico y análisis del entorno, acercándose al artesano a través de sus capacidades y técnica, respetando que pertenecen a realidades completamente distintas sin que por esto intente transformarlas para hacerlas símil a la del diseñador.

Ambos consideran que el diseñador puede intervenir de manera positiva, enriqueciendo y actualizando el trabajo del artesano, invitándolo a hacerlo de un modo distinto que le puede resultar de mayor provecho. Es necesario establecer objetivos y alcances claros, para que no se creen falsas expectativas en cuanto a la colaboración.

Para Mariana Arzate el aprendizaje que recibe el diseñador de estas experiencias no sólo incrementa sus conocimientos en cuanto a historia, comunidades, recursos naturales y procesos, sino que lo sensibiliza, lo abre hacia otras maneras de pensar, vivir y percibir un mismo territorio. Por otro lado, para el artesano estas experiencias lo acercan hacia procesos de creatividad e innovación, la labor está en que ellos puedan comprender cómo emplearlos en distintos niveles.

Al proponer innovaciones en la elaboración de algunas de sus piezas de diseño, diversos artesanos realizan partes del proyecto que posteriormente son unidas para conformar un todo. Para Mariana Arzate el reto más grande es poder incidir en la economía local y por lo tanto en el bienestar de los artesanos.

La forma de convencer a los artesanos para que realicen sus diseños ha sido sensibilizándose a la participación, ya que a veces representan un cambio drástico a lo que usualmente elaboran, el convencimiento se da planteando los beneficios que esto trae consigo, por lo tanto, la reacción al ver el resultado final en la mayoría de los casos, resulta positiva. 
Poder comunicar a los consumidores la fusión que integran sus productos, entre lo artesanal y las nuevas tecnologías, es uno de los elementos esenciales para mejorar las prácticas de consumo responsable. El enfoque de centrarse en la calidad como vehículo para el mejoramiento y una mayor apreciación del trabajo artesanal, es igualmente compartido por el diseñador Víctor Alemán, el cual ha llevado las habilidades y conocimientos de los artesanos y expertos en oficios a su máximo potencial. A continuación, ofrece su opinión sobre algunos cuestionamientos planteados en el cuestionario. ${ }^{4}$

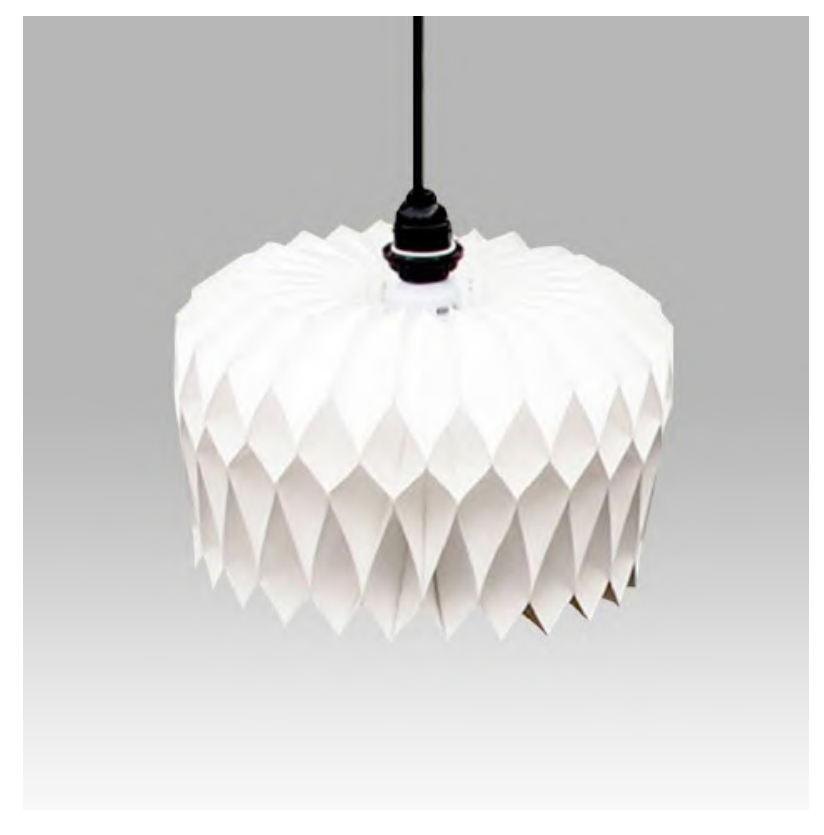

Figura 3. Lámparas Luciérnagas. Foto: archivo Rituales Contemporáneos

\section{Víctor Alemán}

Víctor Alemán se ha destacado por combinar procesos de desarrollo digital con la producción de oficios artesanales locales. Sus piezas se distinguen por su complejidad formal. Esta mezcla de procesos lo ha llevado a desarrollar relaciones profundas con artesanos, siempre con la disposición de ir a aprender, que es el modo en que Alemán considera se debe 
acercar un diseñador, sin miedo a involucrarse y metiendo las manos para ensuciarse. Para Alemán en los proyectos de colaboración con artesanos, el diseñador no tiene tanto que enseñarle al artesano, al contrario el diseñador es el que va a aprender de un especialista.

La responsabilidad del diseñador es poner en la mesa oportunidades, su función es de consejero, su entendimiento sobre el mercado final le permite ayudar al artesano a expandir su gama de productos. Es inútil que el diseñador proponga hasta que no haya aprendido por lo menos teóricamente sobre el oficio, por lo que tiene que respetar el oficio y dedicación de la persona que lo ejerce.

No se debe olvidar que la artesanía es reflejo de un esfuerzo productivo, por lo que entender la realidad social puede ser realmente más significativo que entender procesos productivos. El diseñador aprende a contactarse directamente con las personas que crean los productos, es indispensable que reconozca la transformación técnica y metodológica, para a partir de ahí proponer nuevas aplicaciones. Cuando el diseñador logra entender la complejidad del sistema, puede hacer modificaciones para transgredir los límites de la técnica del artesano. El artesano se beneficia de estas interacciones al ampliar técnicas y métodos, al encontrar que su trabajo puede diversificar su campo de utilidad y por lo tanto también su desarrollo creativo.

Alemán tiene experiencia impartiendo talleres sobre innovación en la creación de productos artesanales, debido a estas experiencias es considera que su educación lo ayudó en cuanto al entendimiento de los métodos de transformación/producción, pero lo que realmente lo ha ayudado a innovar a partir de procesos artesanales, es saber observar y experimentar para poder prever los posibles resultados.

Según la experiencia de Alemán los artesanos tienen una perspectiva muy diferente en cuanto a los productos, a diferencia de los diseñadores. El artesano concibe los objetos que produce son símbolos que se han transmitido de generación en generación, lo que hace que las variaciones en éstos sean casi nulas. Alemán se ha percatado sobre la dificultad 
que tiene un artesano mayor para trabajar en nuevos proyectos, ya que en muchos casos su memoria muscular producto de toda una vida laboral los traiciona, realizando el mismo tipo de piezas.

Uno de los obstáculos que ha afrontado es que no se atrevían a fabricar sus diseños debido a la complejidad formal, una de las maneras en las que se negaban era elevando los costos para que no le conviniera fabricarlo. A partir de estas experiencias es como fue formando sus tácticas para que se animaran a ejecutarlo, una de ellas es desglosar y planear sus proyectos, es decir, presentar pasos individuales a cada especialista.

Un ejemplo de esto es su columpio Mua, el cual para su realización depende de varios procesos en los cuales se involucran maestros herreros y tejedores de mimbre; a los maestros herreros sólo se les presenta una estructura pre-ensamblada, la cual tienen que soldar en cuatro partes, esto es todo lo que ellos ven sobre el proyecto. Por otra parte al maestro tejedor de mimbre se le presenta una estructura que requiere seguir patrones de tejido que ellos ya dominan, esto permite simplificar la complejidad de su trabajo.

Cuando diseña un producto la primera persona en ver el objeto es el artesano que lo construirá y al que Alemán se refiere como el asesor de proyecto. Después se modifica el producto hasta que el artesano sea capaz de imaginar cómo lo creará, una vez que el diseño ya llega a ese nivel de integración, se comienza con el proceso de prototipos en el que se analizan las mejoras necesarias para su facilidad productiva. La reacción que los artesanos han tenido una vez que finalizan el producto diseñado es de sorpresa al observar la escala de los productos con los que han trabajado.

Ya sea tanto para el artesano como para el diseñador, lo mejor que pueden hacer es conformar equipos, entendiendo que no se puede hacer todo solo. Para Alemán el crecimiento se logra participando en una relación ganar/ganar, encontrando personas especializadas en cada una de las áreas. 


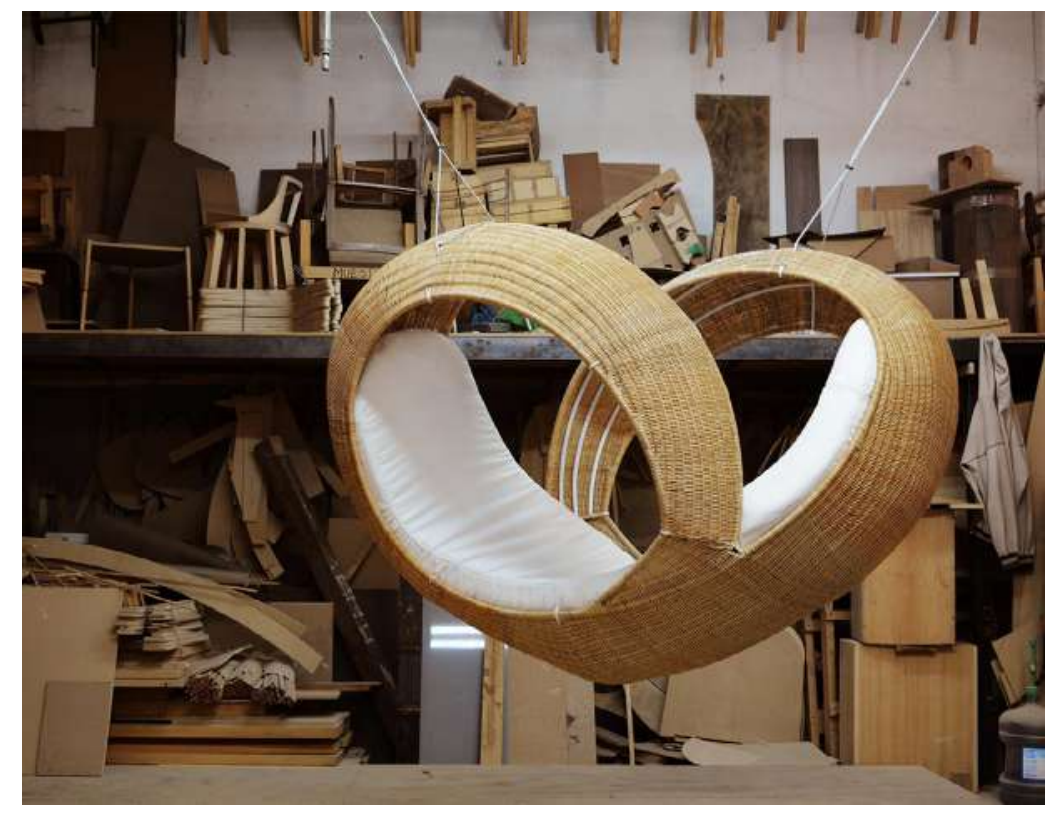

Figura 4. Columpio MUA. Foto: archivo Víctor Alemán.

\section{Hoy tradición, ayer innovación}

Los objetos artesanales, como lo señala Indrasen Vencatachellum (s.f.), director del programa de artes, artesanía y diseño de la UNESCO, tienen una conectividad entre el pasado, el presente y el futuro; lo que hoy es considerado tradición ayer fue innovación, por lo tanto estos objetos son cambiantes y se han transformado a lo largo del tiempo no por moda, sino por una evolución histórica.

Para Vencatachellum los objetos artesanales, al no estar obsesivamente estandarizados como los industriales, adquieren características únicas a través de sus propias limitantes productivas, esto no tiene que ver con demeritar su calidad, simplemente no pertenecen a un sector cuyas reglas se basan en la estandarización obsesiva. La falta de precisión no quiere decir que el objeto no cumple una función específica, y mucho menos estética; de igual manera que no por ser concebidos de manera industrial son funcionales ni estéticos, por lo cual no compiten entre ellos. Lo mismo pasa con los objetos creados por diseñadores, ya sea de modo artesanal o de modo industrial, el buen diseño y el mal diseño se dan en ambas ramas. 


\section{La construcción de un lenguaje contemporáneo en el diseño mexicano}

Lo propio y lo adoptado

Al fragmentar todo lo que se considera ser, se hace un desglose de los aspectos que deveIan la naturaleza sobre la procedencia que conforma ese todo. Esta particularidad proviene de dos fuentes que son las que parten del ser individual y aquellas que se adquieren como parte de un colectivo. Reconocerse como parte de un contexto es el punto de partida desde el cual un diseñador comienza a utilizar sus impulsos creativos para transformar su realidad y la de otros. Poder comprender la idea que una comunidad o un artesano tiene de sí mismo y contrastarla con otras realidades a las que el diseñador tiene acceso, es un manera de poner un proyecto colaborativo en perspectiva y equilibrio.

La identificación con el otro y su entorno es fundamental para que el diseñador extraiga aquellos elementos de autenticidad que hablan de los valores compartidos entre la comunidad y la relación que él mismo haya establecido con el artesano. La brecha que existe entre el mundo del artesano y el del diseñador es parte de lo que Daniel Abadie (2008) menciona en cuanto al destiempo que existe entre ciudades y comunidades, y cómo esto hace que no pueda existir una identidad única, sino que ésta se construye con las elecciones que se realizan individualmente en relación al grupo social al que se pertenece.

Si bien el diseño encuentra sus bases en una serie de momentos, conocimientos y metodologías, la identidad nacional en cuanto al diseño que representa a los países, está determinada por aquellos objetos y servicios que son producto de la creatividad local. Esta identidad está fundada en aspectos como la historia, los materiales locales, las posibilidades tecnológicas, productivas y la educación.

Al hacer tangibles las características fundamentales de un entorno a través de objetos, éstos adquieren distintivos con respecto a otros. Los elementos que se descifran son los que posibilitan identificar y asociar su procedencia, se pueden observar en detalles como la forma, el color, los gráficos y materiales, pero también pueden ser mediante su proceso de elaboración o incluso el propósito de su diseño. 
Algunos países han logrado destacar las características de su diseño de manera muy evidente: los alemanes han hecho que sus productos se destaquen por su funcionalidad y durabilidad; los italianos por su gran calidad en la elaboración de objetos de lujo; los escandinavos por la simpleza, funcionalidad y aprovechamiento de recursos naturales.

Algunas cosas que resalta Guy Julier (2010) al respecto son dos fenómenos, primero la desigualdad que trae la globalización en cuanto a que cada vez más ciudades de primer mundo se convierten en ejes creativos, lo cual provoca un distanciamiento entre las economías basadas en el conocimiento y aquellas manufactureras, esto se ejemplifica con la localización de los estudios de diseño de algunas de las compañías más importantes, los cuales cada vez están más alejados físicamente de los centros de producción de sus proyectos, contribuyendo a la homogeneidad cultural que atrae la globalización.

\section{El diseñador como intérprete de la tradición}

Roger Bartra (2013) plantea la idea de que modernizar la cultura ya pasó, refiriéndose a que los cambios se dan en ciclos; la modernización de la cultura no es algo que se pueda marcar como una etapa pasada, debería de estar sucediendo todo el tiempo. En México aún no hemos alcanzado una conciencia plena sobre el potencial benéfico del diseño, el desaprovechamiento éste y el aferrarse al pasado, es lo que hace que se siga alimentando la imagen cliché del país, mostrando un concepto de lo nacional distorsionado.

El modo más natural de traer a la actualidad aquellas tradiciones que tanto se aprecian es pasándolas por el crisol del trabajo que realizan artesanos, artistas y diseñadores.

A veces pareciera que, en cuanto a temas como la tradición, sólo existen dos tipos de posturas, los que están a favor de conservar la tradición tal cual y los que creen que su naturaleza es evolutiva. Si se apuesta por la segunda, al igual que Juan Acha (2013) se estará de acuerdo en su argumento sobre cómo las culturas populares se encuentran en la pobreza a pesar de que brindan ritos, mitos y magias, estas características hacen que 
los extranjeros encuentren un aire nostálgico en sus productos, sin embargo, necesitamos sacudirnos esa percepción y superarnos en nuestro propio beneficio cultural. La única forma de lograr esto es rompiendo aquella cápsula del tiempo a la que se han confinado a los productos artesanales.

La profesión del diseñador es optimista, abierta al cambio, a que las cosas siempre pueden ser mejores y que invariablemente siempre hay una solución. El diseñador tiene que ser lo suficientemente positivo o ingenuo para estar convencido que puede cambiar el mundo a partir de su trabajo. La identidad de los diseñadores, se va conformando de miles de referencias cruzadas tanto personales como profesionales, así como experiencias, este capital cultural sirve como inspiración directa para sus creaciones, aunque inevitablemente como en cualquier profesión se comparten referencias, información e ideologías con colegas locales e internacionales.

El diseñador contemporáneo mexicano, planteándolo desde una perspectiva generalizada, aprendió mediante su educación universitaria sobre aquellos esfuerzos que se han hecho a lo largo de la historia por mejorar la vida cotidiana, las bases que hoy dan forma a su labor a través de los tiempos dorados que se vivieron en la Bauhaus, el empuje creativo del ingenio humano mediante las piezas de mobiliario de los Eames, han sido influenciados por los principios del buen diseño de Dieter Rams, pero también por la rebeldía de Philippe Starck; admiradores del trabajo de Ettore Sottsass, Marc Newson, Konstantin Grcic o Jonathan Ive, seguidores de la filosofía de Steve Jobs, pero a la vez, del trabajo realizado por Luis Barragán o Pedro Ramírez Vázquez; interesados en la impresión 3D pero volteando la mirada a los oficios clásicos artesanales.

La educación del diseñador no sólo se da en el aula, sino en los talleres mediante los cuales adquiere experiencia con los materiales, es por esta razón que es capaz de reconocer oportunidades dentro de cualquier espacio que cultive un conocimiento productivo, experimenta un goce al observar el proceso y dentro de su cabeza es inevitable dejar el qué podría hacer él para mejorar, ya sea en cuanto a procesos o características del producto que lo haga más atractivo. 
Parte de la gran ventaja con la que cuenta un diseñador al ejercer en un país como México, es la amplia variedad de pequeños talleres que aún se pueden encontrar por todos lados, hay conocimiento ancestral regado por todas las ciudades y comunidades.

Al encontrar estos nichos de oportunidad, donde además la necesidad de trabajo obliga a la apertura hacia nuevas propuestas, el diseñador reconoce las posibilidades del oficio e identifica la habilidad del artesano que lo desempeña, a partir de esto es como el diseñador se hace consiente del conocimiento detrás de aquellas destrezas, analiza los objetos y es capaz de retomar esa tradición para ajustarla a productos que él, con su conocimiento del entorno y el mercado actual puede adaptar para su venta.

Otras de las interrogantes que se encuentran en los cuestionarios tienen el propósito confrontar y recabar la opinión de los entrevistados para detectar puntos en común y en desacuerdo sobre temas como la reinterpretación y revalorización de la tradición, algunos ejemplos que se podrían seguir y si consideran que hay un lenguaje en común. ${ }^{5}$

Para que un diseñador sea capaz de reinterpretar la tradición, Alejandra Villegas del CDO considera que lo se puede esperar de un diseñador es que sea capaz de entender el contexto y el origen de las tradiciones, que pueda comprender los símbolos, significados y la identidad, esto le lleva tiempo, el proceso puede ser largo. Sin embargo, el diseñador a diferencia del artesano es el que tiene el conocimiento sobre las demandas del mercado. La mayor amenaza hacia la tradición, según Villegas, no son las tendencias que dicta el mercado, sino el trabajo irresponsable de no entender el contexto. Cuando un concepto es impuesto al artesano lo más probable es que no lo entienda y no logre identificarse, por lo que parte del trabajo del diseñador es lograr que ellos traduzcan aquellas tendencias desde sus propios valores interpretativos.

5 Cfr. Alemán, V. (15 de julio de 2014). Entrevista Tesis. (A. Rojo, Entrevistador). Recuperado de: drive.google.com/ open?id=1ntzGbO3u6mWeSEpKoKu1y9ApfBMjZtWu Arzate, M. (23 de noviembre de 2014). Entrevista Tesis. (A. Rojo, Entrevistador). Recuperado de: drive.google.com/open?id=1oeCiqZof1DISeSTQSNiWGaJ4EiDIFfPY De la O, J. (12 de agosto de 2014). Entrevista Tesis. (A. Rojo, Entrevistador). Recuperado de: drive.google.com/open?id=1eNrgc8BwNBhBlxq-vCOsvRvym0UAgp7P Villegas, A. (21 de abril de 2014). Entrevista tesis CDO. (A. Rojo, Entrevistador). Recuperado de: drive.google.com/open?id=1ZplUxQFgn0JpeLbIW6SV9thzMv7aqdlg 
José de la O es más puntual, para él lo que hace que un diseñador pueda reinterpretar la tradición es simplemente apropiársela. El estudiar diseño no le resulta fundamental para tener el deseo de hacer algo bien y diferente, para lograr innovar considera que se necesitan simplemente tener ganas de que el trabajo transcienda.

En cambio para Rituales Contemporáneos la formación profesional del diseñador sí es pilar para que logre reinterpretar los objetos y procesos. Para ellos el mercado y sus tendencias no rivalizan en lo absoluto con la tradición.

Alemán al igual que Villegas, coincide en que para que el diseñador reinterprete la tradición necesita hacer uso de su capacidad de análisis, cuestionarse el cómo y el porqué de los objetos. Para Alemán el proceso previo al boceto de un objeto es esencial, ya que ahí es cuando se les da sentido. Para él no se debe olvidar que las artesanías a fin de cuentas siguen siendo productos y como tal tienen que adaptarse al mercado para poder evolucionar.

\section{La revalorización de la tradición como un método para el desarrollo de un diseño mexicano contemporáneo}

Los países considerados como referentes a seguir en cuanto al desarrollo de una identidad propia en el diseño local a través de la reinterpretación de las tradiciones, varían. Para Alejandra Villegas y Rituales Contemporáneos los escandinavos siguen siendo un ejemplo al desarrollar su diseño a través de sus elementos comunes; los finlandeses han logrado que el diseño sea una parte de su identidad como pueblo, encontrando un eje común de inspiración a partir de la carencia de luz que experimentan debido a largos inviernos y reinterpretándola con el uso de materiales naturales y colores de gran intensidad.

En cuanto a la resignificación de sus tradiciones, Japón es un referente constante para los cuatro entrevistados anteriormente, aunque para Alemán el caso de este país no podría ser replicable en México, ya que la cultura es muy distinta a la nuestra, además de que su sistema exalta otras virtudes en sus artesanías; ellos han aprendido a adaptar sus objetos al 
presente según la estructura del país y su tradición, han respetado los cánones de creación, pero sobre todo han entendido las ventajas de la transformación de la técnica.

Para José de la O, Holanda y Dinamarca tienen un estilo muy propio, e incluso Estados Unidos gracias al renacimiento de lo hecho a mano. Sin embargo, tanto para él como para Alemán en nuestro caso, seguir ejemplos no es lo idóneo, deberíamos de estar observando detenidamente qué se necesita cambiar y hacerlo; México necesita descifrar su propia combinación de factores que propicien la creación de objetos con identidad cultural. Esta búsqueda sólo se puede realizar a través del trabajo diario, el resultado no se puede acelerar es continuo, generacional y constante.

Más allá de si se pueden replicar modelos o si se cuenta con los apoyos necesarios, el diseño mexicano ahí está y ahí ha estado desde hace mucho tiempo, se puede decir que cuenta con una identidad, aunque no esté definida al 100\% y no haya sido construida con ese propósito explícito. México actualmente se ha ido construyendo a la par del lastre de vivir comparando el presente con el pasado, presente que además muchas veces sale perdiendo porque al parecer siempre hubo tiempos mejores que por supuesto a nadie menor de 50 años le tocó vivirlos. Estas ideas un tanto nostálgicas y un tanto retrogradas aún rondan, por lo que el desprenderse de aquellas creencias es un acto de rebeldía que todo creador actual enfrenta.

La incertidumbre de no saber en qué etapa nos encontramos en cuanto a la construcción de una identidad en el diseño mexicano, hace que no se entienda si ya pasó, si se encuentra en construcción, o si ya se va en el tercer intento. Lo que queda claro es que esto no se ha definido. Para Villegas, estamos en una etapa de búsqueda, en la que se está reinventando el diseño, los proyectos colaborativos con artesanos es uno de los caminos, los diseñadores han estado en continua experimentación con materiales y técnicas artesanales edificando puntos en común en cuanto a la identidad. 
Al ser procesos largos y complejos, sería apresurado asegurar que ahí está la identidad del diseño mexicano, esa evaluación se va a hacer con los años para así saber si estos proyectos llegaron para quedarse.

Rituales Contemporáneos coincide con Villegas, aunque no creen que todas las piezas producto del diseño mexicano contribuyen a la definición de una identidad, sin embargo sí en que hay una constante búsqueda, la cual se ha ido construyendo a partir de sus antecedentes en la cultura popular y tradiciones.

De acuerdo con Alemán, una cualidad del diseño mexicano es la variedad de propuestas formales y simbólicos que conjunta. Para él lo que aún no se alcanza es la mezcla en perfecta medida de rasgos históricos que influyen nuestro presente, esto es evidente al observar cómo los objetos mexicanos encajan en otros contextos; ya que muchos diseñadores han tomado los lenguajes de producción de otras culturas y se han apropiado de estos interviniéndolos.

Por su parte, De la O ve el diseño como una manifestación cultural de su sociedad, tanto en lo bueno como en lo malo. Los esfuerzos que realizan los diseñadores aun teniendo muchas condiciones en contra y una carencia de reconocimiento a su labor sobrepasa la sociedad en la cual están inmersos.

Para incluir a la sociedad no basta con los esfuerzos realizados por el gremio, se necesita del apoyo del gobierno y de instituciones de todo tipo, poniendo el ejemplo al dar la confianza a creadores mexicanos para desarrollar proyectos. Este empuje se tiene que traducir en apoyo a la difusión sobre el diseño, sus potencial, beneficios y alcances en el mejoramiento de la vida de todos. La integración del diseño a los programas gubernamentales es una cuestión clave para su primer entendimiento por parte de funcionarios quienes podrían maximizar esa difusión. 
La prueba más fehaciente de que esta revalorización de la tradición por iniciativa de los propios diseñadores existe, siguen siendo las exposiciones y los eventos comerciales itinerantes como los mercados de diseño donde se pueden adquirir los productos.

Los esfuerzos que ha realizado el gremio del diseño en México, eventualmente contribuyen a obtener resultados a largo plazo. Si bien el nombramiento de la Ciudad de México como Capital del diseño 2018 es un gran avance, todavía se necesita trabajar en traducir esto a la vida diaria, a que las empresas mexicanas hagan uso de éste en todos niveles.

A pesar de que evidentemente muchos de los eventos y exhibiciones emergen de intenciones positivas, sin proponérselo estos esfuerzos tienden a caer en un círculo vicioso de promoción y convencimiento dentro del mismo grupo de personas que ya están inmersos en el tema. Se puede encontrar a las mismas personas en los mismos eventos, comentando los unos con los otros, sería interesante poder lograr que esta información permee hacia otros sectores en mayor medida.

En cuanto a la difusión del diseño, para De la O el diseño necesita tomar un rol más intelectual, necesita ser cuestionado y favorecer los ejercicios de crítica. Esto es evidente en la mayoría de los medios impresos especializados en diseño, los cuales se convierten en reseñas de tendencias y eventos.

Por otro lado, la existencia de blogs y revistas especializadas, es parte de lo que Rituales Contemporáneos considera como un aspecto positivo que están alentando a un cambio, así como las exhibiciones y la creación del Directorio de Diseño Mexicano. Sin embargo, resaltan igualmente la necesidad de que el diseño llegue a las personas, que sea partícipe de su vida cotidiana, y que sea utilizado en las empresas mexicanas para generar beneficios. Alemán se enfoca en la transición de pasar de ser creativos a ser creadores, esto en el sentido de que el ingenio mexicano ha permitido solucionar problemas con lo que se tiene a la mano en el momento; pero el cambiar a ser creadores generaría más contenido y por lo tanto un mayor éxito del diseño como disciplina en cualquier proyecto. 
El uso de las exposiciones como un medio de difusión mediáticamente resulta efectivo, aunque no siempre con los resultados esperados. Es un recurso por el cual pasa tanto el trabajo de artesanos como el de los diseñadores, su objetivo, que pareciera obvio, en muchos casos si no tiene la difusión adecuada corre el riesgo de acabar siendo más un mecanismo de simulación que una situación que haya proporcionado mejoras drásticas. Esto no quiere decir que no estén cumpliendo una función de divulgación del conocimiento y trabajo nacional, y mucho menos que sean poco importantes, pero se requiere encontrar caminos que hagan que sean visitadas por una mayor diversidad de personas.

El espacio museístico siempre es complejo y de apreciación subjetiva, aún hay públicos que les sigue pareciendo extraño encontrar en un museo mesas, sillas o cualquier otro objeto cotidiano; observar una vasija de barro negro colocada dentro de un capelo, aunque el objeto podría encontrarse de igual manera en un mercado de artesanías.

Del mismo modo, puede resultar extraño ver grupos de estudiantes de diseño viendo una exposición de algún diseñador que se ha hecho de prestigio haciendo piezas disfuncionales, es decir fuera del credo que se repite cuando se pasa por una educación de diseño. Todas esas posibilidades se encuentran dentro de un mismo espacio y son producto de diversas propuestas y visiones sobre una temática.

Una de las exposiciones más reciente referente a la artesanía y diseño, es la exposición de Ida y Vuelta / Diseño contemporáneo en México, montada en 2014 en el Centro Nacional de las Artes por la curadora Ana Elena Mallet. Esta muestra fue una revisión del diseño nacional desde su forma de producción actual. Se presentaron piezas elaboradas resultado de producciones artesanales e industriales. Lo que determina el diseño mexicano se plantea a través de tres líneas de investigación con el fin de mostrar los materiales, las técnicas manuales y prácticas artesanales a través de los cuales los diseñadores y artesanos innovan y desarrollan nuevos lenguajes que generan identidad. 
Los ejes temáticos que se propusieron fueron:

- Una mirada local

- Retomando el oficio

- Diseño social

En Una Mirada Local se encuentran piezas elaboradas de manera local; retomando materiales y técnicas pasadas para revivirlas en elementos nuevos. En Retomando el Oficio, se discute la mezcla de procesos que los diseñadores actuales emplean entre el trabajo manual artesanal y procesos semi industriales, apoyándose en los oficios locales.

Por último Diseño Social, plantea la tendencia a hacer uso del diseño como un agente de cambio, mediante proyectos colaborativos generando nuevos modelos de identidad. Hubiera sido interesante contar con el espacio suficiente para que los proyectos detrás de los resultados pudieran ser explicados.

Sin embargo, este ejemplo de conjunción del trabajo de diseñadores actuales, permite entender el proceso que se está viviendo y cómo está sustentado en revalorar lo local. En todas las piezas se encuentra en síntesis, un gran mestizaje de muchos elementos resueltos en nuevas propuestas plurales.

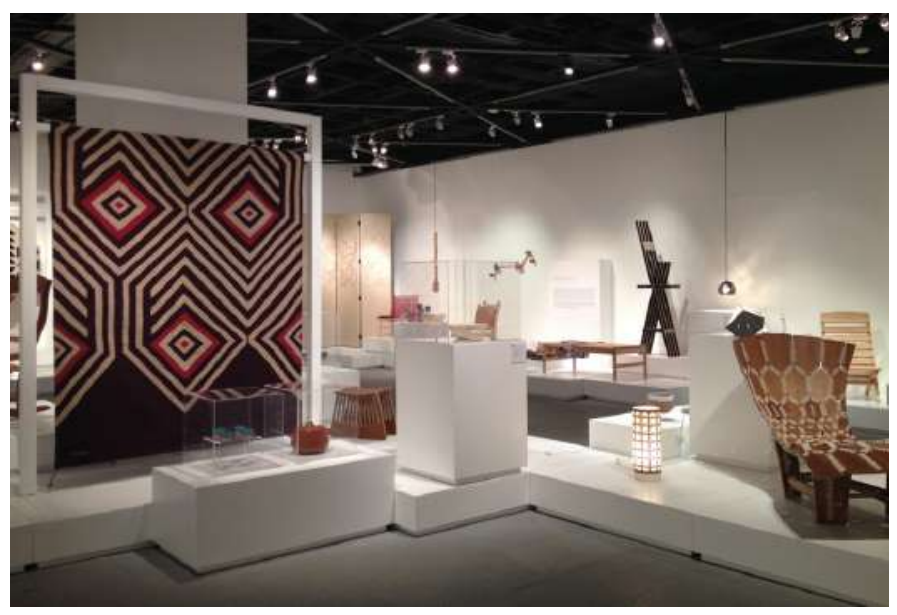




\section{Conclusiones}

El desarrollo de las industrias creativas representa una gran área de oportunidad para el crecimiento del país, aquellos involucrados en este sector están acostumbradas a impulsar y difundir sus propios trabajos, por lo que un mayor empuje por parte de instituciones gubernamentales y educativas haría una gran diferencia en poco tiempo. La valoración de los recursos creativos resultante de los encuentros entre diseñadores y artesanos es esencial para que estas relaciones se sigan dando, que el artesano tenga trabajo y los diseñadores logren la reinserción de estos productos al mercado de manera exitosa.

El diseñador al adentrarse en estos proyectos aprende a base de ensayo y error lo que puede hacer o cambiar para mejorar el aprendizaje. El salir del ensimismamiento de su labor diaria, le permite encontrar nuevas formas de transformar el trabajo que se viene haciendo desde generaciones atrás; al compartir con el artesano la información a la cual tienen acceso.

El diseño transita sus propios problemas, la falta de atención hacia éste radica en el desentendimiento de la actividad y sus alcances. Una mejor y efectiva difusión del diseño en el país podría impulsar su uso en más empresas. La única manera que tiene el diseño para lograr convencer es a partir de los resultados de su trabajo, por lo que necesita de oportunidades para hacerlo. Si México se considera un país innovador tiene que poner atención en los conocimientos y productos que emergen localmente; en parte esto se podría lograr propiciando la evolución de los productos artesanales, sacándolos de su confinamiento, transformándolos, propiciando su evolución más que su conservación.

En cuanto a la importancia de la intervención del diseñador, se comprueba que su participación es benéfica para la mejora del objeto artesanal, no porque el trabajo de sociólogos o antropólogos no sea un gran apoyo, sino porque el diseñador entiende la práctica y tiene los conocimientos para transformarla de manera tangible, entiende los procesos y al objeto, por lo tanto, puede descifrarlo. 
A través de los ejemplos de proyectos contemporáneos, se obtuvo un panorama sobre la diversidad y naturaleza de los esfuerzos realizados por distintos actores, queda claro que ninguno tenía bases precisas, las han ido implementando a base de su propia experiencia. Para lograr estos proyectos se involucran personal y profesionalmente, permitiéndoles entender sus propias limitaciones y virtudes.

Sobre el cuestionamiento a los proyectos revisados sobre la identidad del diseño mexicano, queda claro que ninguno se encuentra seguro en qué consiste y si esto se ha logrado. La identidad del diseño mexicano no ha sido construida como parte de un plan o de una marca nacional, carece de estructura y coherencia, sin embargo, esto otorga libertad a los creadores nacionales a seguir encontrándola a través de su propio trabajo. La prevención de la homogeneidad cultural de nuestra época, se puede combatir dando oportunidades a los diseñadores y creadores nacionales, ya que mediante su trabajo fungen como mediadores entre lo local y lo foráneo.

La identidad del diseñador mexicano, al igual que la de cualquier otro, está formada por múltiples referencias, aunque existe un denominador común entre los diseñadores nacionales que es el aprovechamiento de los materiales y oficios locales, más aún en un país semi industrializado, aunque no por eso, se encuentren alejados de las nuevas tecnologías, sino que simplemente los procesos productivos se facilitan mediante este sistema.

Los fundadores de los proyectos contemporáneos apuestan porque México encuentre la manera de reconciliarse con sus tradiciones a través del diseño y sin replicar tal cual las acciones de otros países, apuestan por la unión del gremio para promover más ejercicios de crítica que hagan que el diseño tome un rol más intelectual, que se haga partícipe a la sociedad y la industria.

Sobre la difusión a través de recursos como las exposiciones, se demuestra que las propuestas sobre el tema artesanía y diseño están bien logradas, sin embargo, el gran problema es que esta información llega en su mayoría a las mismas personas que ya la tenían, no por falta de motivación, sino que se requiere de mayor difusión fuera de los espacios comunes de siempre. 


\section{Referencias}

Acha, J. (2013). Aproximaciones a la identidad latinoamericana. Ciudad de México: Trillas.

Alemán, V. (2013). Victor Alemán Studio. Recuperado el 2014, de http://www.victoraleman.mx/index.php/ product/furniture

Alemán, V. (15 de Julio de 2014). Entrevista Tesis.

(A. Rojo, Entrevistador)

Arzate, M. (23 de noviembre de 2014). Entrevista Tesis. (A. Rojo, Entrevistador)

Bartra, R. (2013). Oficio Mexicano. Ciudad de México: Debolsillo.

Borges, A. (2011). Design+Craft The Brazilian path. Sao Paulo: Terceiro Nome.

Centro de Diseño de Oaxaca. (7 de noviembre de 2014). Centro de diseño de Oaxaca. Recuperado el 2 de diciembre de 2014, de http://cdo.gob.mx/esp/ binomios-creativos-que-es/

CONEVAL. (2013). Informe de evaluación específica 2012-2013, FONART. Ciudad de México.

Craft Revival Trust, UNESCO. (2005). Encuentro entre artesanos y diseñadores. Guía práctica.

De la O, J. (2013). The chair that rocks. Recuperado el 4 de marzo de 2013, de thechairthatrocks.com

De la O, J. (Dirección). (2013). The chair that rocks [Película]. México https://vimeo.com/60749319

De la O, J. (12 de Agosto de 2014). Entrevista Tesis. (A. Rojo, Entrevistador)

Diez, A. (2013). Conversación con Indrasen Vencatachellum

FONART. (2009). Diagnóstico de la capacidad de los artesanos en pobreza para generar ingresos sostenibles. Ciudad de México.
Frías, J. (diciembre de 2012). Policy innovación design. Recuperado el 12 de agostol de 2014, de http://www.seeplatform.eu/map

Hvid, K. (15 de febrero de 2015). Design to improve life. Obtenido de http://designtoimprovelife.dk/moveok-great-creative-industries-denmark/

Julier, G. (2010). Más allá de las fronteras: Historia del diseño, transnacionalización y globalización. En I. Campi, R. Pelta, A. Calvera, V. Narotzky, M. Freixa, \& C. Bayo, Diseño e historia. Tiempo, lugar y discurso. Ciudad de México: Designio.

Sennett, R. (2000). La corrosión del carácter. Barcelona, España: Anagrama.

UNESCO (2001) El reconocimiento de excelencia para la artesanía. Recuperado el 8 julio de 2014, de http://portal.unesco.org/culture/es/ev.phpURL_ID =37649\&URL_DO=DO_TOPIC\&URL_ SECTION=201.html

UNESCO (2013). Creative economy report 2013 UNESCO. Recuperado el 8 julio de 2014 unesco.org/culture/pdf/creative-economyreport-2013.pdf

UNESCO (2014). Artesanía diseño. Recuperado el 8 julio de 2014, de www.unesco.org/es/guia-industriasculturales

Villegas, A. (21 de abril de 2014). Entrevista tesis CDO. (A. Rojo, Entrevistador)

Williams, R. (11 de febrero de 2014).

The guardian. Recuperado el 4 de enero de 2015, de http://www.theguardian.com/ artanddesign/2014/feb/11/design-craft-subjectsdecline-in-schools 